\title{
Effect of spatial coherence of LED sources on image resolution in holographic displays
}

Vahid Pourreza Ghoushchi, Mehdi Aas, Erdem Ulusoy, Hakan Ürey

Vahid Pourreza Ghoushchi, Mehdi Aas, Erdem Ulusoy, Hakan Ürey, "Effect of spatial coherence of LED sources on image resolution in holographic displays," Proc. SPIE 10126, Advances in Display Technologies VII, 101260A (16 February 2017); doi: 10.1117/12.2252413

SPIE. Event: SPIE OPTO, 2017, San Francisco, California, United States 


\title{
Effect of spatial coherence of LED sources on image resolution in holographic displays \\ Vahid Pourreza Ghoushchi ${ }^{*}$, Mehdi Aas ${ }^{\mathrm{b}}$, Erdem Ulusoy ${ }^{\mathrm{a}}$, Hakan Ürey ${ }^{\mathrm{a}}$ \\ ${ }^{a}$ Optical Microsystems Laboratory (OML), Koç University, Istanbul, Turkey; \\ ${ }^{\mathrm{b}}$ Complex Photonic Systems (COPS), University of Twente, Enschede, The Netherlands.
}

\begin{abstract}
Holographic Displays (HDs) provide 3D images with all natural depth cues via computer generated holograms (CGHs) implemented on spatial light modulators (SLMs). HDs are coherent light processing systems based on interference and diffraction, thus they generally use laser light. However, laser sources are relatively expensive, available only at some particular wavelengths and difficult to miniaturize. In addition, highly coherent nature of laser light makes some undesired visual effects quite evident, such as speckle noise, interference due to stray light or defects of optical components. On the other hand, LED sources are available in variety of wavelengths, has small die size, and no speckle artifact. However, their finite spatial size introduce some degree of spatial incoherence in an HD system and degrade image resolution, which is the subject of the study in this paper. Our theoretical analysis indicates that the amount of resolution loss depends on the distance between hologram and SLM image planes. For some special configurations, the source size has no effect at all. We also performed experiments with different configurations using lasers and LEDs with different emission areas that vary from 50 $\mu \mathrm{m}$ to $200 \mu \mathrm{m}$, and determined Contrast Transfer Function (CTF) curves which agree well with our theoretical model. The results show that it is possible to find configurations where LEDs combined with pinholes almost preserve natural resolution limit of human eye while keeping the loss in light efficiency within tolerable limits.
\end{abstract}

Keywords: Holographic Displays, Computer Generated Holograms, CGH, Spatial Coherence, Image Resolution. Coherence Effect, Digital Holography, Coherence Effects in Resolution.

\section{INTRODUCTION}

Holography, invented in 1947 by Dennis Gabor, is a method that employs diffraction and interference for recording and reconstruction of optical wavefronts [1]. In Computer Generated Holography (CGH) approach fringe patterns are calculated by a computer and then optical wavefronts are reconstructed by a Spatial Light Modulator (SLM) [2-4]. Holographic Displays (HDs) are able to generate real or virtual 3D images without requiring 3D glasses for the viewer $[5,6]$. Holographic displays have emerging applications in augmented reality and virtual reality [7-9].

Holographic displays rely on diffraction and interference and use high coherence laser illumination that produce adverse side effects such as speckle noise. Since presence of speckle noise severely degrades reconstructed images quality, this issue remains one of the fundamental challenges in digital and optical holography. Different optical methods (such as reducing the spatial coherence of light sources) [10] and computational methods (digital image processing and CGH computation algorithms) $[11,12]$ have been implemented for speckle reduction. Use of Light Emitting Diodes (LEDs) make it possible to reduce the undesirable interference effects and speckle noise in holographic displays to a certain extent at the expense of reduced resolution. Illuminating the SLM surface with low coherence LEDs results in smoother images in comparison to high coherence laser sources.

\footnotetext{
*Email: vghoushchi13@ku.edu.tr
}

Advances in Display Technologies VII, edited by Liang-Chy Chien, Tae-Hoon Yoon, Sin-Doo Lee, Proc. of SPIE Vol. 10126, 101260A - @ 2017 SPIE - CCC code: 0277-786X/17/\$18 · doi: 10.1117/12.2252413 
LEDs as sources in digital holography, have previously been investigated in holographic microscopy [13] and effect of partial spatial coherence in digital holographic microscopy was also studied $[14,15]$.

The usage of LEDs as incoherent light sources for holographic displays have gained popularity. Full-color holographic displays using LEDs as sources with phase-only SLMs and amplitude-only SLMs has been proposed [4, 16-18]. Color head-mounted holographic displays that employ LED illumination has been developed to overcome the common vergence-accommodation conflict in 3D Head Mounted Displays[19, 20]. A new multiplexing method and a calibration technique for correcting the misaligned wavefronts due to multiplexing in color HDs has been studied [21, 22]. The influence of spatial and temporal coherence on digital holographic reconstructions has been studied[23, 24]. The effect of low coherence LED illumination in optical holographic reconstruction has been investigated[25].

In this paper we investigate the effect of source spatial coherence on the resolution of the reconstructed holograms. Our theoretical analysis indicates that the degradation in image resolution due to spatial incoherence of the light source depends on the optical architecture of the holographic display, and in particular depends on the distance between the apparent object and SLM planes. We also present experiments and verify our theoretical model. A reflective phase-only Liquid Crystal on Silicon (LCOS) SLM (Holoeye PLUTO-VIS-014) was illuminated with a red LED to reconstruct the holograms. We used $54 \mu \mathrm{m}, 107$ and $205 \mu \mathrm{m}$ pinholes in front of the LED to increase its spatial coherence. SLM is located in different locations and SLM image planes are placed at various planes. Our experimental results strongly support our theoretical analysis.

\section{ANALYSIS}

Our optical setup is shown in figure 1. Diverging light from an LED source is collimated by a lens and illuminates the reflective phase-only spatial light modulator. The modulated light passes once again through the lens (which now acts as a Fourier transform lens) and reaches a beam splitter. Rays reflected by the beam splitter and reach a diaphragm, an imaging lens and a CCD camera, on which the holograms are reconstructed. The LED source is used with pinholes of varying sizes to control the extent of spatial incoherence of the source. A He-Ne laser coupled to a single mode fiber is also used to serve as a reference source with almost perfect spatial coherence. For a particular object point, the CGH on the Spatial Light Modulator in general becomes an off-axis lens term that converts the collimated illumination wave to a new wave that becomes equivalent to the wave emitted by the object point upon passage through the Fourier lens.

When the source has perfect spatial coherence and when the hologram on the SLM is appropriately calculated, one gets the holographic reconstruction at the camera plane with the best resolution. However, when the source has a nonzero extent, additional oblique plane wave components illuminate the SLM and form shifted replicas of the reconstruction at the CCD plane which are superimposed on the actual desired reconstruction, leading to a loss in spatial resolution. The extent of degradation, however, depends on the configuration of the system. This is best understood by considering the image of the SLM formed by the Fourier lens and the imaging lens. If the plane where this image forms coincides with the CCD plane, then all reconstructions are exactly aligned with each other, and the spatial incoherence of the source has no effect on the resolution at all. However, if there is a certain distance between the SLM image plane and the CCD plane, reconstructions due to oblique waves do not overlap, and lead to a blurring effect on the reconstructed image. The degree of blurring increases as the distance between the mentioned planes increase. Therefore, resolution degradation due to source incoherence in general depends on the optical configuration and the location of the reconstruction plane. 


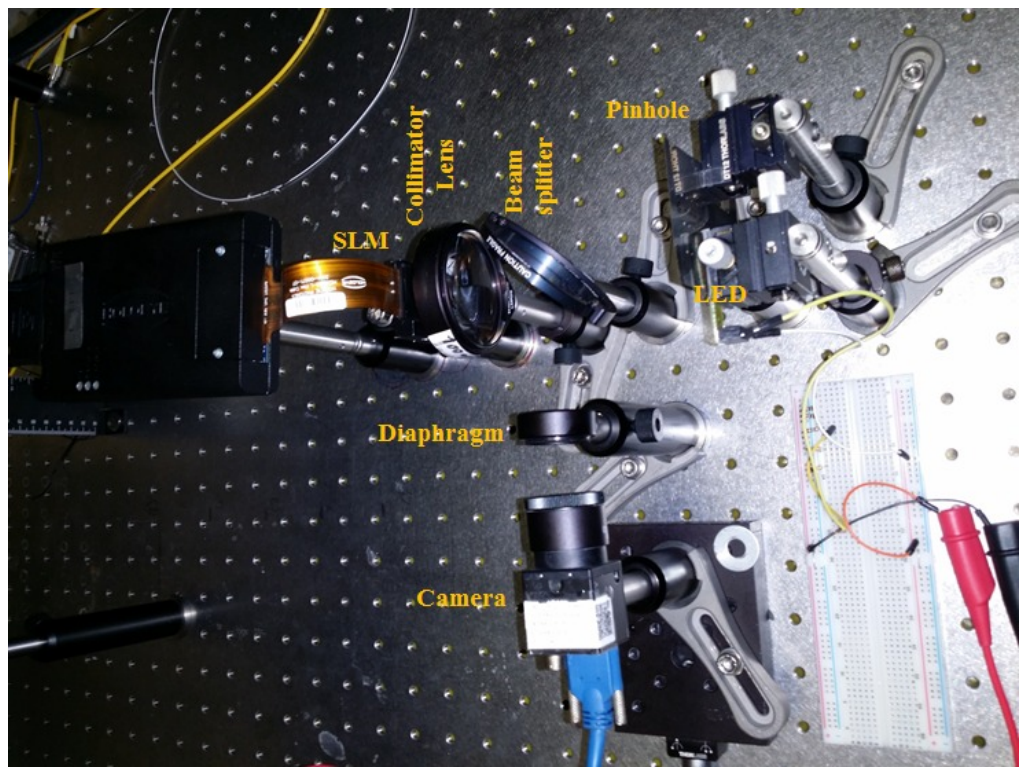

Figure 1. The Optical Setup With an incoherent LED source: Light generated by an LED passes through a pinhole to increase its spatial coherence, and then gets collimated by a lens and illuminates the surface of a spatial light modulator. Beam modulated and reflected backwards from the SLM passes through the same lens, gets reflected by a beam splitter and passes through a diaphragm that serves as a Fourier filter. Finally after passing through an imaging lens the holograms are reconstructed at the CCD plane of a camera.

In our experiment, we choose the focal length of the Fourier lens as $100 \mathrm{~mm}$, and we fixed the distance between the Fourier lens and imaging lens to $100 \mathrm{~mm}$. We study five different configurations where the SLM to Fourier lens distance is taken as $20,40,60,80,100 \mathrm{~mm}$. The focal length of the imaging lens is $50 \mathrm{~mm}$. For each of the five cases, we placed the CCD camera (hologram reconstruction plane) at a distance of $50 \mathrm{~mm}, 55.5 \mathrm{~mm}$ and $63.3 \mathrm{~mm}$. Straightforward analysis shows that only in three cases (SLM to Fourier lens: $60 \mathrm{~mm}$, imaging lens to CCD: $63.3 \mathrm{~mm}$, SLM to Fourier lens: $80 \mathrm{~mm}$, imaging lens to CCD: $55.5 \mathrm{~mm}$, SLM to Fourier lens: $100 \mathrm{~mm}$, imaging lens to CCD: $50 \mathrm{~mm}$ ) the SLM image is formed on the reconstruction plane, whereas in other cases, there is a difference in between. In terms of percentage difference between reconstruction and SLM image planes, 15 configurations can be categorized into 5 groups, as follows: Group 1, the group mentioned above. In this group best images are expected regardless of source coherence. Group 2 involves five cases: SLM to lens: $40 \mathrm{~mm}$ and CCD is at $63.3 \mathrm{~mm}$, SLM to lens distance is $60 \mathrm{~mm}$ and CCD is at $55.5 \mathrm{~mm}$, SLM to lens distance is $80 \mathrm{~mm}$ and CCD can be at both $63.3 \mathrm{~mm}$ and $50 \mathrm{~mm}$, SLM to lens distance is $100 \mathrm{~mm}$ and CCD is at $55.5 \mathrm{~mm}$. Group 3 has four possibilities including: CCD is at $63.3 \mathrm{~mm}$ and SLM to lens distances are $20 \mathrm{~mm}$ and $100 \mathrm{~mm}$, CCD is at $55.5 \mathrm{~mm}$ and SLM to lens distance is $40 \mathrm{~mm}$, CCD is at $50 \mathrm{~mm}$ and SLM to lens distance is $60 \mathrm{~mm}$. Group 4 has two cases: SLM to lens distance is $20 \mathrm{~mm}$ and CCD at $55.5 \mathrm{~mm}$, SLM to lens distance is $40 \mathrm{~mm}$ and CCD at $50 \mathrm{~mm}$. Finally in Group 5 we have the only case of SLM to lens distance $20 \mathrm{~mm}$ and CCD at 50 $\mathrm{mm}$. As the group number increases, the difference between SLM image and CCD planes increase, and we expect the degradation in resolution to get worse.

\section{EXPERIMENTAL RESULTS}

The image of the 1951 USAF resolution chart was reconstructed by the laser and LED sources with different emission areas. Applying pinholes in front of the LEDs we control the degree of source spatial coherence which increases as the pinhole size is decreased. For each group discussed in the previous section, reducing the spatial coherence of the sources results in reduction in reconstructed holograms quality, except from Group 1. On the other hand, for the high coherence laser source we expect to obtain the same quality for all SLM to lens distances and imaging lens to CCD distance combinations. Figure 2 shows cases in which the SLM to lens distances are 60 
$\mathrm{mm}$ and the CCD is $63.3 \mathrm{~mm}$ away from the imaging lens (Group 1) for a) a laser source, b) a red LED source with $54 \mu \mathrm{m}$ pinhole size, c) a red LED with $107 \mu \mathrm{m}$ pinhole size and d) a red LED with $205 \mu \mathrm{m}$ pinhole size. This is one of the three particular combinations where the source extent or spatial coherence does not affect the quality of the constructed holograms. On the other hand, figure 3 indicates a case in which the SLM to lens distances are $20 \mathrm{~mm}$ and the CCD is $50 \mathrm{~mm}$ away from the imaging lens for a) a laser source, b) a red LED source with $54 \mu \mathrm{m}$ pinhole size, c) a red LED with $107 \mu \mathrm{m}$ size pinhole size and d) a red LED with $205 \mu \mathrm{m}$ pinhole size. In these cases the difference between the CCD plane and SLM image plane is highest. Therefore, the resolution of the reconstructed holograms is seriously reduced by the increasing LED sizes.
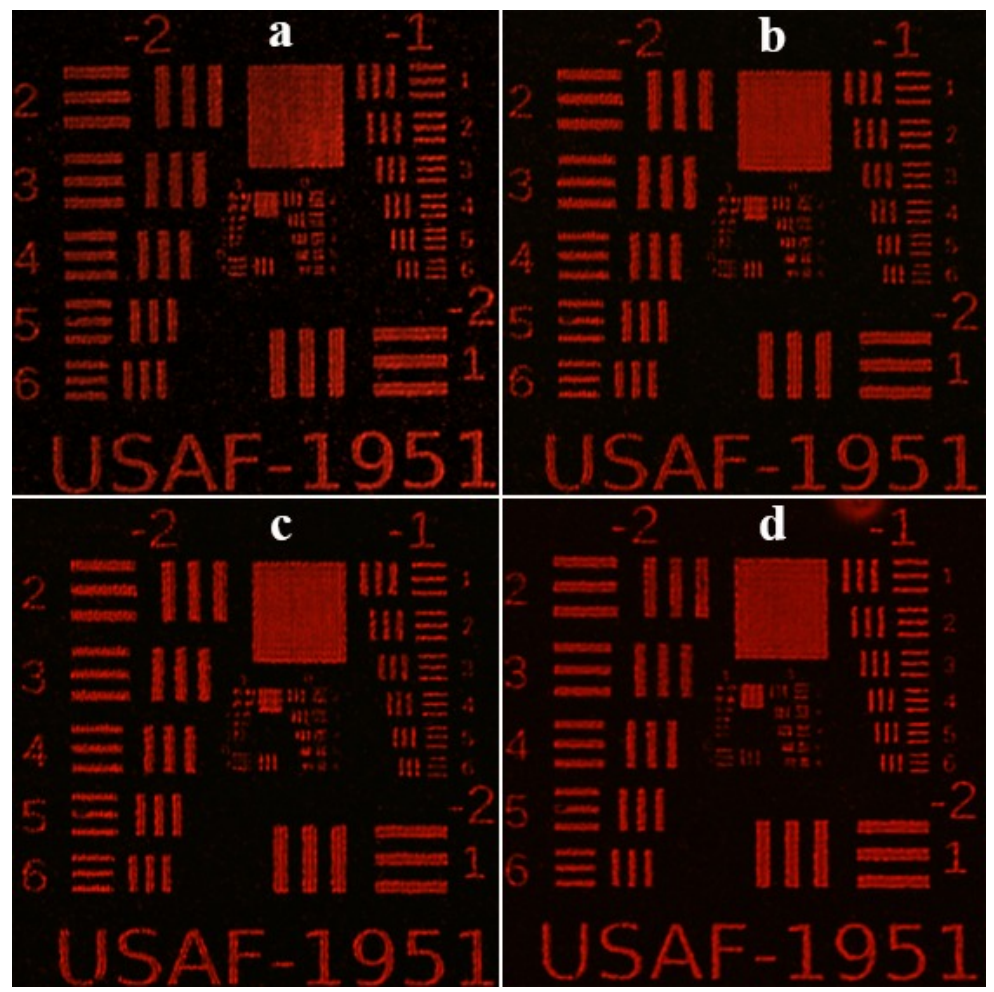

Figure 2. Reconstructed images of USAF resolution target when SLM to lens distance is $60 \mathrm{~mm}$ and the CCD to imaging lens distance is $63.3 \mathrm{~mm}$ for a)a laser source b)a red LED source with $54 \mu \mathrm{m}$ pinhole size c)a red LED with $107 \mu \mathrm{m}$ pinhole size and d)a red LED with $205 \mu \mathrm{m}$ pinhole size. 

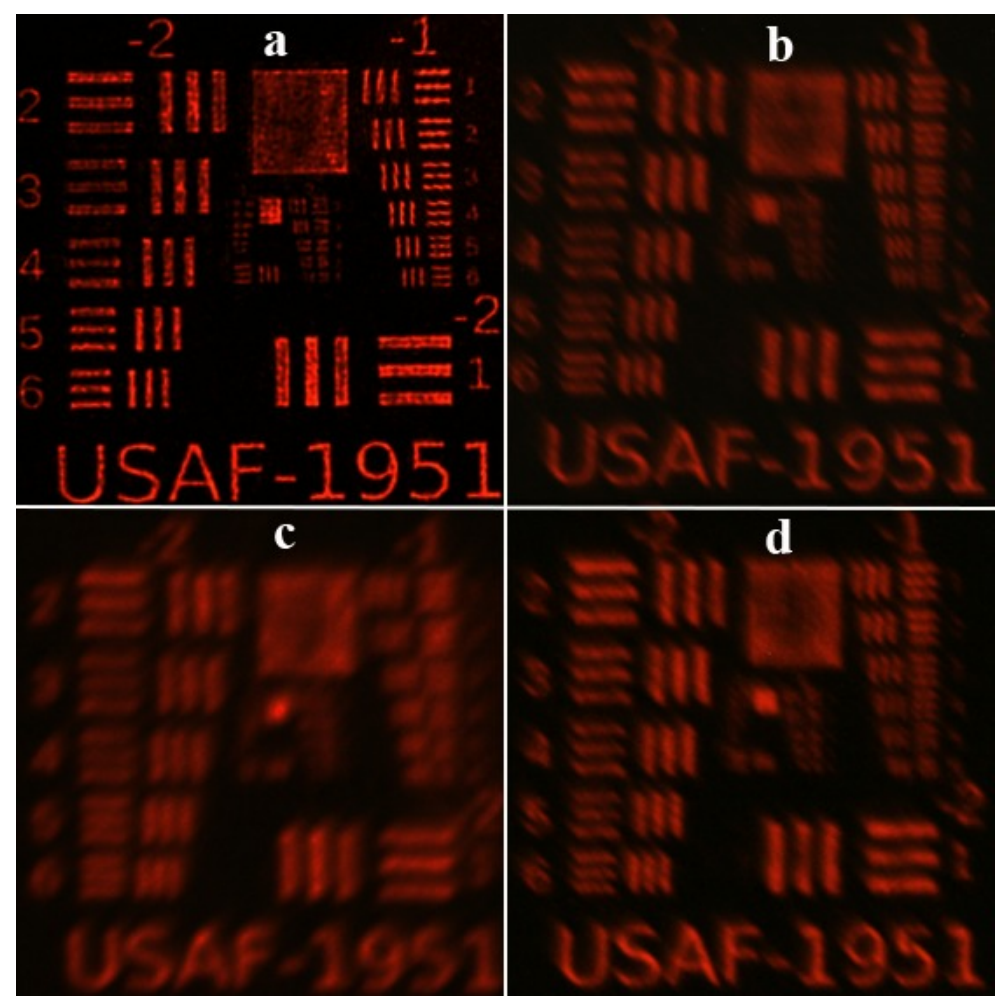

Figure 3. Reconstructed images of USAF resolution target when SLM to lens distance is $20 \mathrm{~mm}$ and the CCD to imaging lens distance is $50 \mathrm{~mm}$ a)a laser source b)a red LED source with $54 \mu \mathrm{m}$ pinhole size c)a red LED with $107 \mu \mathrm{m}$ pinhole size and d)a red LED with $205 \mu \mathrm{m}$ pinhole size.

In our experiments, four different light sources were tested on each of 15 different SLM to lens and imaging lens to CCD combinations that were categorized in 5 groups. The data is analyzed to determine the effect of the light source spatial coherence on the quality of the reconstructed images. The relation between the spatial coherence of the sources and contrast for different groups is illustrated in figures 4 to 6 in terms of contrast transfer functions. As seen in Figure 4 for group 1 the CTF of the reconstructed images for various sources are almost independent from the spatial coherence of the source, as expected. In these special cases the resolution of the reconstructed images with LEDs are the same with the laser source images even in higher spatial frequencies. Figure 5 shows that the effect of spatial coherence starts to degrade the CTF curve in higher spatial frequencies for group three members in LED sources. These trends show that there is a significant difference between the contrast of the LED source and the laser source. In group 5 the LEDs image contrast, even in low spatial frequencies, drop down sharply in comparison with the high coherence laser source. These results verify that our experimental results agree well with our theoretical analysis. 


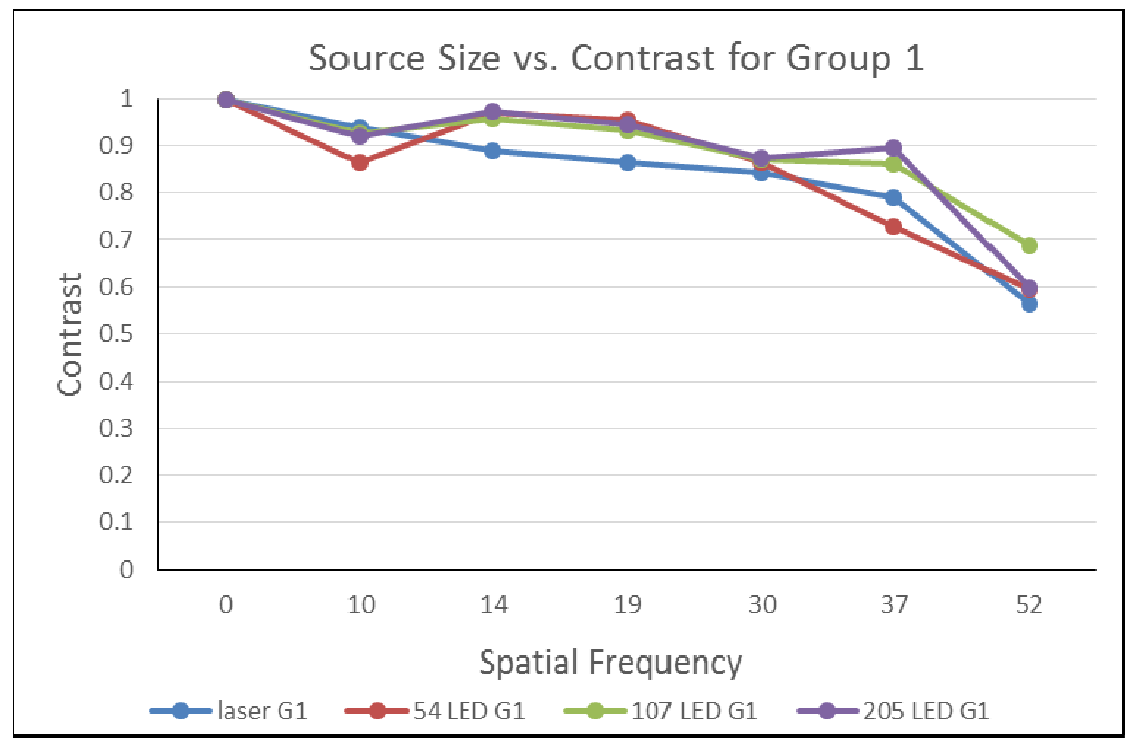

Figure 4. The CTF (Contrast Transfer Function) curves for holographic reconstructions with sources of varying degree of spatial coherence for group 1 configurations (SLM image and hologram reconstruction planes coincide). The blue line indicates the laser source, the red line the LED source with $54 \mu \mathrm{m}$ pinhole size, the green line the LED source with $107 \mu \mathrm{m}$ pinhole size and the purple line shows the LED source with $205 \mu \mathrm{m}$ pinhole size. This figure shows that for group 1 members, the quality of the reconstructed images is almost independent of spatial coherence of the sources.

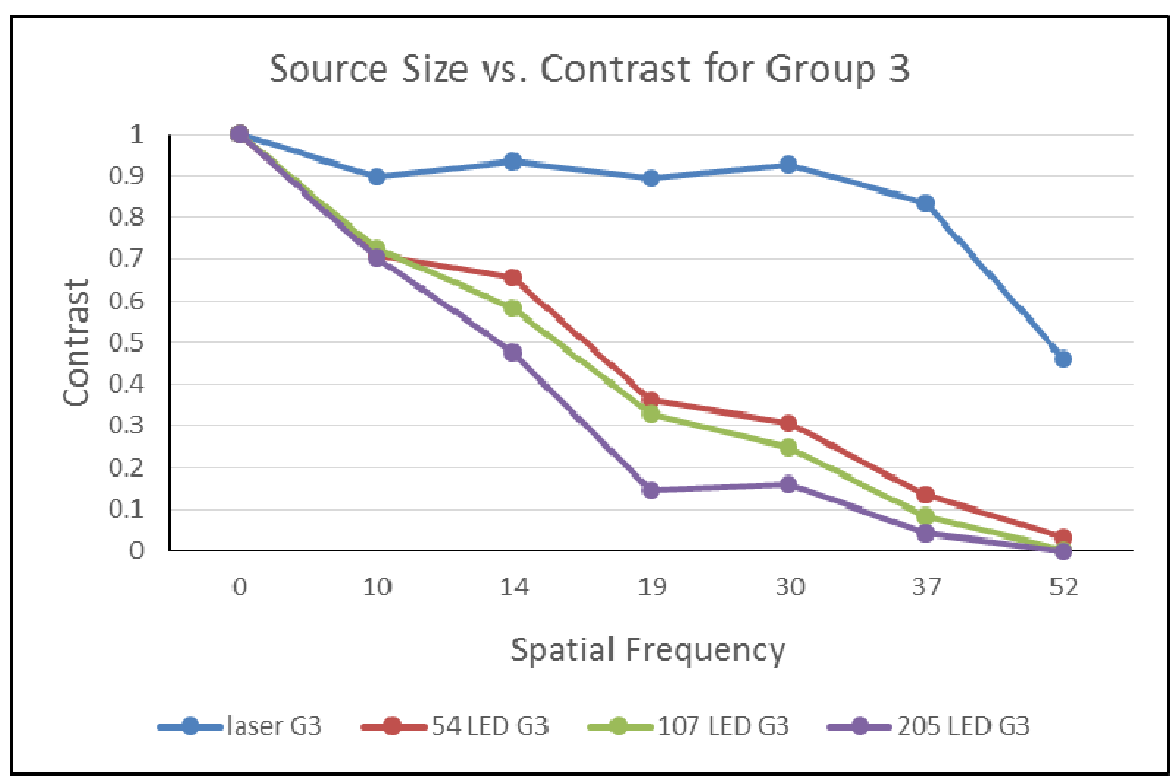

Figure 5. Counterpart of Figure 4 for group 3 configurations (a moderate distance between SLM image and hologram reconstruction planes). This figure shows that as the spatial coherence of LED sources decrease the quality of the reconstructed images decrease accordingly. 


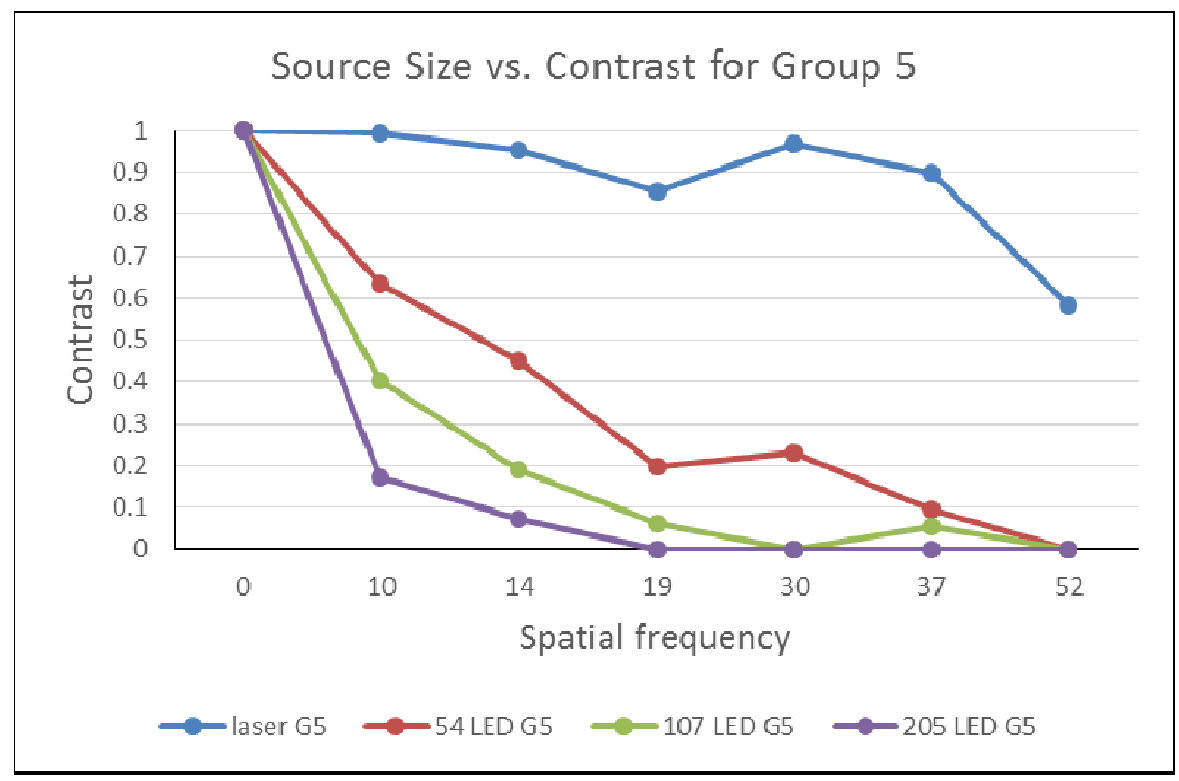

Figure 6. Counterpart of Fig 4 for Group 5 (largest relative distance between SLM image and hologram reconstruction planes). The sensitivity of image quality to source coherence is highest

\section{CONCLUSION}

In this study we investigated the effect of the spatial coherence on the reconstructed holograms contrast. We proposed that the amount of resolution loss depends on the optical configuration of the holographic display, and in particular on the distance between hologram reconstruction plane and SLM image planes. Our experiments and data analysis are in good agreement with our theoretical model. We showed that when hologram reconstruction plane and SLM plane are optically conjugate to each other, the degree of spatial coherence of the source has almost no effect on the resolution of the reconstructed images. By choosing these particular configurations, or by keeping the distance between SLM image and reconstruction planes close, LEDs can be used as sources for holographic displays without incurring significant degradation in resolution.

Acknowledgement: This work is supported by European Research Council under the Seventh Framework Programme (FP7/2007-2013) ERC grant agreement no 340200, with acronym Wear3D.

\section{REFERENCES}

[1] T.-C. Poon, [Digital holography and three-dimensional display: Principles and Applications] Springer Science \& Business Media, (2006).

[2] H. Yoshikawa, and T. Yamaguchi, "Computer-generated holograms for 3D display," Chinese Optics Letters, 7(12), 1079-1082 (2009).

[3] H. Yoshikawa, [Computer-Generated Holograms for White Light Reconstruction] Springer US, Boston, MA(2006). 
[4] X. J. Zhang, X. Liu, and H. F. Li, "Reproduction of a computer-generated hologram with a white light source and liquid crystal device," Optical Engineering, 46(4), 4 (2007).

[5] F. Yaraş, H. Kang, and L. Onural, "State of the Art in Holographic Displays: A Survey," Journal of Display Technology, 6(10), 443-454 (2010).

[6] C. Slinger, C. Cameron, and M. Stanley, "Computer-Generated Holography as a Generic Display Technology," Computer, 38(8), 46-53 (2005).

[7] M. Kujawinska, T. Kozacki, C. Falldorf et al., "Multiwavefront digital holographic television," Optics express, 22(3), 2324-2336 (2014).

[8] K. Takano, and K. Sato, "Color electro-holographic display using a single white light source and a focal adjustment method," Optical Engineering, 41(10), 2427-2433 (2002).

[9] K. Hong, J. Yeom, C. Jang et al., "Full-color lens-array holographic optical element for threedimensional optical see-through augmented reality," Optics letters, 39(1), 127-130 (2014).

[10] M. Tziraki, R. Jones, P. M. W. French et al., "Photorefractive holography for imaging through turbid media using low coherence light," Applied Physics B, 70(1), 151-154 (2000).

[11] J. Garcia-Sucerquia, J. A. H. Ramírez, and D. V. Prieto, "Reduction of speckle noise in digital holography by using digital image processing," Optik - International Journal for Light and Electron Optics, 116(1), 44-48 (2005).

[12] D. Mengu, E. Ulusoy, and H. Urey, "Non-iterative phase hologram computation for low speckle holographic image projection," Optics Express, 24(5), 4462-4476 (2016).

[13] F. Dubois, L. Joannes, and J.-C. Legros, "Improved three-dimensional imaging with a digital holography microscope with a source of partial spatial coherence," Applied Optics, 38(34), 7085-7094 (1999).

[14] F. Dubois, M.-L. Novella Requena, C. Minetti et al., "Partial spatial coherence effects in digital holographic microscopy with a laser source," Applied Optics, 43(5), 1131-1139 (2004).

[15] U. Gopinathan, G. Pedrini, and W. Osten, "Coherence effects in digital in-line holographic microscopy," Journal of the Optical Society of America A, 25(10), 2459-2466 (2008).

[16] F. Yaraş, H. Kang, and L. Onural, "Real-time phase-only color holographic video display system using LED illumination," Applied optics, 48(34), H48-H53 (2009).

[17] K. Takano, and K. Sato, "Full-color electroholographic three-dimensional display system employing light emitting diodes in virtual image reconstruction," Optical Engineering, 46(9), 8 (2007).

[18] W. Harm, A. Jesacher, G. Thalhammer et al., "How to use a phase-only spatial light modulator as a color display," Optics Letters, 40(4), 581-584 (2015).

[19] E. Moon, M. Kim, J. Roh et al., "Holographic head-mounted display with RGB light emitting diode light source," Optics express, 22(6), 6526-6534 (2014).

[20] T. Yoneyama, C. Yang, Y. Sakamoto et al., "Eyepiece-type full-color electro-holographic display for binocular vision." 8644, 864413-864413-8.

[21] T. Kozacki, and M. Chlipala, "Color holographic display with white light LED source and single phase only SLM,” Optics Express, 24(3), 2189-2199 (2016).

[22] W. Zaperty, T. Kozacki, R. Gierwiało et al., "RGB imaging volumes alignment method for color holographic displays." 10031, 1003117-1003117-8.

[23] T. Kozacki, and R. Jozwicki, "Near-field digital hologram registration in partially coherent stationary object illumination." 5484, 437-444.

[24] K. A. Nugent, "Partially coherent diffraction patterns and coherence measurement," Journal of the Optical Society of America A, 8(10), 1574-1579 (1991).

[25] M. Chlipała, and T. Kozacki, "Resolution limits in holographic display with LED illumination." 9527, 95270A-95270A-9. 